\title{
THE APPROXIMATION BY PARTIAL SUMS OF FOURIER SERIES
}

BY

\author{
R. SALEM AND A. ZYGMUND
}

1. Let $f(x)$ be a continuous function having the period $2 \pi$. If this function satisfies a Lipschitz condition of order $\alpha(0<\alpha \leqq 1)$, that is to say if

$$
|f(x+h)-f(x)|=O\left(|h|^{\alpha}\right)
$$

uniformly in $x$ as $h \rightarrow 0$, we shall say briefly that $f$ belongs to Lip $\alpha$. Following a notation already used (see Zygmund [4](1)) we shall say that $f$ belongs to $\operatorname{lip} \alpha, 0<\alpha<1$, if $|f(x+h)-f(x)|=o\left(|h|^{\alpha}\right)$ uniformly as $h \rightarrow 0$.

It is a classical result of Lebesgue (see Zygmund [5, p. 61]; hereafter this book will be denoted by T.S.) that if $f$ belongs to Lip $\alpha$ and if $s_{n}$ denotes the $n$th partial sum of the Fourier series of $f$, then, uniformly

$$
\left|s_{n}(x)-f(x)\right|=O\left(n^{-\alpha} \log n\right)
$$

and it is known that the logarithm in the second member cannot be omitted.

The question whether the last relation can be sharpened by the suppression of the logarithm if, in addition to the hypothesis that $f$ belongs to Lip $\alpha$, we suppose also that $f$ is of bounded variation seems to have been overlooked and it is the main purpose of this paper to give the solution of this problem. It will be sufficient to consider the case $0<\alpha<1$ since if $\alpha=1$ it is well known that the relation (1) can not be sharpened (see T.S. pp. 61-62, examples 6 and 8).

Let $a_{0} / 2+\sum_{1}^{\infty}\left(a_{n} \cos n x+b_{n} \sin n x\right)$ be the Fourier series of a continuous function of bounded variation, $f(x)$ of period $2 \pi$. It is clear that such a function can not be monotonic in $(0,2 \pi)$ since $f(0)=f(2 \pi)$; but we shall say that $f(x)$ belongs to the monotonic type if the Fourier-Stieltjes series

$$
d(f+C x) \sim C+\sum_{1}^{\infty}\left(n b_{n} \cos n x-n a_{n} \sin n x\right)
$$

is, for some $C$, the Fourier-Stieltjes series of a monotonic function; in other words if there exists a constant $C$ such that $f(x)+C x$ is either never decreasing or never increasing in $(-\infty,+\infty)$.

Our results can now be stated in the following two theorems:

THEOREM I. If the continuous function $f(x)$ of period $2 \pi$ belongs to Lip $\alpha$ $(0<\alpha<1)$ and is of monotonic type, then

Presented to the Society, September 17, 1945; received by the editors May 9, 1945.

(1) Numbers in brackets refer to the literature cited at the end of the paper. 


$$
\left|s_{n}(x)-f(x)\right|=O\left(n^{-\alpha}\right)
$$

uniformly in $x$, where $s_{n}(x)$ is the nth partial sum of the Fourier series of $f$.

THEOREM II. The preceding theorem is no longer true if instead of supposing that $f(x)$ is of monotonic type we suppose that $f(x)$ is of bounded variation. More precisely, for every positive $\alpha$ less than 1 there exists a continuous function $\phi(x)$ of bounded variation belonging to Lip $\alpha$ and such that for arbitrarily large values of $n$

$$
\max \left|s_{n}(x)-\phi(x)\right|>\frac{\log n}{\omega(n) \cdot n^{\alpha}}
$$

$\omega(n)$ being a function increasing infinitely with $n$, as slowly as we please.

Proof of Theorem I. Suppose, to fix the ideas, that there is a constant $C$ such that $F(x)=f(x)+C x$ is increasing. Denoting as usual by $D_{n}(t)$ the Dirichlet's kernel sin $((2 n+1) t / 2)(2 \sin t / 2)^{-1}$ we have

$$
\pi\left[s_{n}(x)-f(x)\right]=\int_{0}^{\pi}[f(x+t)+f(x-t)-2 f(x)] D_{n}(t) d t .
$$

Since $f \in \operatorname{Lip} \alpha$, it is obvious that

$$
\int_{0}^{\eta}[f(x+t)+f(x-t)-2 f(x)] D_{n}(t) d t=O\left(n^{-\alpha}\right)
$$

uniformly in $x$, if $\eta=O(1 / n)$. To study the integral from $\eta$ to $\pi$, we observe that it is equal to

$$
\int_{\eta}^{\pi}[F(x+t)+F(x-t)-2 F(x)] D_{n}(t) d t
$$

and we split it into integrals of the type $\int_{\pi / 2^{k}}^{\pi / 2}$ where $k$ runs from 1 to the integral part of $\log n / \log 2$. By the second mean value theorem, the integral in the interval $\left(\pi / 2^{k}, \pi / 2^{k-1}\right)$ is equal to

$$
\begin{aligned}
\frac{1}{2 \sin \left(\pi / 2^{k+1}\right)} \int_{\pi / 2^{k}}^{\xi}[F(x+t)+F(x-t)-2 F(x)] \sin \left(\frac{2 n+1}{2} t\right) d t \\
\\
\left(\frac{\pi}{2^{k}}<\xi<\frac{\pi}{2^{k-1}}\right)
\end{aligned}
$$

and since $F(x+t)+F(x-t)-2 F(x)$ is an increasing function of $t$ an application of the second mean value theorem shows that this expression is

$$
O\left[\left(\frac{2^{k}}{\pi}\right)\left(\frac{\pi}{2^{k}}\right)^{\alpha} \frac{1}{n}\right]=O\left[\frac{2^{(1-\alpha) k}}{n}\right]
$$


uniformly in $x$. Thus the integral (2) is

$$
O\left[\frac{1}{n} \sum_{k=1}^{m} 2^{(1-\alpha) k}\right]
$$$$
m=\left[\frac{\log n}{\log 2}\right]
$$

that is to say

$$
O\left[\frac{1}{n} 2^{(1-\alpha) m}\right]=O\left(n^{-\alpha}\right)
$$

which proves Theorem I. Clearly if we start from the assumption $f \in \operatorname{lip} \alpha$ we prove in the same way $\left|s_{n}(x)-f(x)\right|=o\left(n^{-\alpha}\right)$.

An easy consequence of Theorem I is that we have $\left|s_{n}(x)-f(x)\right|=O\left(n^{-\alpha}\right)$ whenever $f(x)$ is continuous, of bounded variation, and its total variation $V(x)$ belongs to $\operatorname{Lip} \alpha(0<\alpha<1)$.

(An elementary example of a continuous function of bounded variation whose total variation does not admit the same modulus of continuity as the function itself is provided by the function $x^{\beta} \sin 1 / x(1<\beta<2)$ which belongs to $\operatorname{Lip}(\beta / 2)$ and whose total variation can not belong to $\operatorname{Lip} \alpha$ for $\alpha>\beta-1$. The proof is left to the reader.)

Proof of Theorem II. Let $\alpha$ be given $(0<\alpha<1)$. For every positive integer $n$ we consider the function continuous and of period $2 \pi$ defined as follows

$$
\begin{aligned}
& f_{n}(x)=\frac{1}{n^{\alpha}} \sin n x \text { for } 0 \leqq x \leqq \frac{\left[n^{\alpha}\right] \pi}{n}, \\
& f_{n}(x)=0 \quad \text { for } \quad \frac{\left[n^{\alpha}\right] \pi}{n} \leqq x \leqq \pi, \\
& f_{n}(-x)=f_{n}(x)
\end{aligned}
$$

where $\left[n^{\alpha}\right]$ denotes as usual the integral part of $n^{\alpha}$. We write $\left[n^{\alpha}\right] \pi / n=\lambda_{n}$.

The total variation of $f_{n}(x)$ in $(-\pi,+\pi)$ is

$$
2 \int_{0}^{\lambda_{n}} n^{1-\alpha}|\cos n x| d x<2 n^{1-\alpha} \lambda_{n}<2 \pi \text {. }
$$

Moreover, $f_{n}(x)$ belongs to Lip $\alpha$ uniformly in $n$. For, if $x_{1}, x_{2}$ belong to the interval $\left(0, \lambda_{n}\right)$, we have

$$
f_{n}\left(x_{2}\right)-f_{n}\left(x_{1}\right)=\frac{1}{n^{\alpha}} 2 \sin n \frac{x_{2}-x_{1}}{2} \cos n \frac{x_{2}+x_{1}}{2} ;
$$

thus, if $\left|x_{2}-x_{1}\right| \geqq 1 / n$, we have

$$
\left|f_{n}\left(x_{2}\right)-f_{n}\left(x_{1}\right)\right| \leqq \frac{2}{n^{\alpha}} \leqq 2\left|x_{2}-x_{1}\right|^{\alpha} .
$$

On the other hand, if $\left|x_{2}-x_{1}\right|<1 / n$, we have 


$$
\left|f_{n}\left(x_{2}\right)-f_{n}\left(x_{1}\right)\right|<\frac{2}{n^{\alpha}} \cdot \frac{n\left|x_{2}-x_{1}\right|}{2}<\frac{\left|x_{2}-x_{1}\right|}{\left|x_{2}-x_{1}\right| 1-\alpha}=\left|x_{2}-x_{1}\right|^{\alpha} .
$$

Thus, taking into account that $f_{n}(x)$ is everywhere continuous, even, and vanishes in $\left(\lambda_{n}, \pi\right)$, we deduce that $\left|f_{n}\left(x_{2}\right)-f_{n}\left(x_{1}\right)\right| \leqq 4\left|x_{2}-x_{1}\right| \alpha$, for any two points of the interval $(-\pi, \pi)$.

Finally, let us note that

$$
\int_{0}^{\pi} f_{n}(t) \frac{\sin n t}{t} d t=\frac{1}{n^{\alpha}} \int_{0}^{\lambda_{n}} \frac{\sin ^{2} n t}{t} d t=\frac{1}{n^{\alpha}} \int_{0}^{\left[n^{\alpha}\right] \pi} \frac{\sin ^{2} v}{v} d v>A \frac{\log n}{n^{\alpha}},
$$

$A$ being a constant depending on $\alpha$ only, and that

$$
\begin{aligned}
\int_{0}^{\pi} f_{n}(t) \frac{\sin q t}{t} d t & =\frac{1}{n^{\alpha}} \int_{0}^{\lambda_{n}} \frac{\sin n t \sin q t}{t} d t \\
& =\frac{1}{n^{\alpha}} \int_{0}^{\lambda_{n}} \frac{\sin ^{2}(n+q) t / 2}{t} d t-\frac{1}{n^{\alpha}} \int_{0}^{\lambda_{n}} \frac{\sin ^{2}(n-q) t / 2}{t} d t \\
& =\frac{1}{n^{\alpha}} \int_{\lambda_{n}|n-q| / 2}^{\lambda_{n}|n+q| / 2} \frac{\sin ^{2} v}{v} d v>0 .
\end{aligned}
$$

Let now $\omega(n)$ be a function increasing infinitely with $n$ as slowly as we please. Let $\left\{n_{k}\right\}$ be a sequence of integers such that

$$
\sum \frac{1}{\omega\left(n_{k}\right)}<\infty \text {. }
$$

Consider the function

$$
\phi(x)=\sum_{k=1}^{\infty} \frac{f_{n_{k}}(x)}{\omega\left(n_{k}\right)} .
$$

$\phi(x)$ is continuous, of period $2 \pi$, of bounded variation, and belongs to Lip $\alpha$. If $s_{n}(x)$ denotes the $n$th partial sum of its Fourier series, we have

$$
s_{n}(0)=\frac{1}{\pi} \int_{0}^{\pi} \phi(t) \frac{\sin n t}{t} d t+O\left(\frac{1}{n}\right) .
$$

Hence, by the preceding remarks,

$$
s_{n_{k}}(0)>\frac{A}{\pi} \frac{\log n_{k}}{\omega\left(n_{k}\right) \cdot n_{k}^{\alpha}}-O\left(\frac{1}{n_{k}}\right)
$$

which is the same as

$$
\left|s_{n_{k}}(0)-\phi(0)\right|>C \frac{\log n_{k}}{\omega\left(n_{k}\right) n_{k}^{\alpha}}
$$

$C$ being a positive constant, and thus proves our theorem. 
2. In this section, which is not connected with the preceding one, we collect some other results concerning mainly the order of magnitude of $s_{n}-f$ and on the order of $s_{n}$, as compared with the corresponding expressions for the conjugate series.

THEOREM III. Let $f(x)$ be a continuous function of period $2 \pi$, and $s_{n}(x)$ the partial sum of order $n$ of its Fourier series. Let $\bar{f}(x)$ be the function conjugate to $f$, and $\xi_{n}(x)$ its Fourier series partial sum of order $n$. If $f(x)-s_{n}(x)=O\left(n^{-\alpha}\right)$ uniformly in $x(\alpha>0)$, then $\bar{f}(x)-\bar{s}_{n}(x)=O\left(n^{-\alpha}\right)$ uniformly in $x$.

Suppose first that $0<\alpha<1$. Then, by a well known theorem (see de la Vallée Poussin [3, p. 57]), $f(x)$ being approximable by trigonometric polynomials of order $n$ with an error of order $n^{-\alpha}, f(x)$ belongs to Lip $\alpha$. Hence, by Privaloff's theorem (see T.S. p. 156), $\bar{f}(x)$ belongs also to Lip $\alpha$.

This implies, by S. Bernstein's theorem (see T.S. p. 62),

$$
\begin{aligned}
& \sigma_{n}(x)-f(x)=O\left(n^{-\alpha}\right), \\
& \bar{\sigma}_{n}(x)-\bar{f}(x)=O\left(n^{-\alpha}\right)
\end{aligned}
$$

uniformly in $x$, where $\sigma_{n}$ and $\bar{\sigma}_{n}$ denote Fejér's sums of order $n$ of $f(x)$ and $\bar{f}(x)$ respectively.

By (1) and by our hypothesis $s_{n}(x)-\sigma_{n}(x)=O\left(n^{-\alpha}\right)$. Hence, by S. Bernstein's theorem on the maximum of the derivative of a trigonometric polynomial (see T.S. p. 155)

$$
\frac{s_{n}^{\prime}(x)-\sigma_{n}^{\prime}(x)}{n+1}=O\left(n^{-\alpha}\right),
$$

the primes denoting derivatives with respect to $x$.

Writing $s_{n}=A_{0}+A_{1}+\cdots+A_{n}$, where $A_{n}=a_{n} \cos n x+b_{n} \sin n x$, and $\jmath_{n}=B_{1}+B_{2}+\cdots+B_{n}$, where $B_{n}=a_{n} \sin n x-b_{n} \cos n x$, we have

$$
s_{n}-\sigma_{n}=\frac{A_{1}+2 A_{2}+\cdots+n A_{n}}{n+1} \text {. }
$$

Hence (3) gives

$$
\frac{B_{1}+2^{2} B_{2}+\cdots+n^{2} B_{n}}{(n+1)^{2}}=O\left(n^{-\alpha}\right) .
$$

Denoting now by $\bar{\sigma}_{n}^{2}$ the Cesàro mean of the second order of $\bar{f}$, we have

$$
\begin{aligned}
& \bar{\sigma}_{n}=\frac{n B_{1}+(n-1) B_{2}+\cdots+B_{n}}{n+1}, \\
& \bar{\sigma}_{n}^{2}=\frac{n(n+1) B_{1}+(n-1) n B_{2}+\cdots+1 \cdot 2 B_{n}}{(n+1)(n+2)},
\end{aligned}
$$


so that

and

$$
\bar{\sigma}_{n}-\bar{\sigma}_{n}^{2}=\frac{n B_{1}+2(n-1) B_{2}+\cdots+n \cdot 1 \cdot B_{n}}{(n+1)(n+2)}
$$

$$
\begin{aligned}
\bar{s}_{n}-\bar{\sigma}_{n}-\frac{n+2}{n+1}\left(\bar{\sigma}_{n}-\right. & \left.\bar{\sigma}_{n}^{2}\right) \\
= & \frac{(n+1) B_{1}+(n+1) 2 B_{2}+\cdots+(n+1) n B_{n}}{(n+1)^{2}} \\
& -\frac{n B_{1}+(n-1) 2 B_{2}+\cdots+n \cdot 1 \cdot B_{n}}{(n+1)^{2}} \\
= & \frac{B_{1}+2^{2} B_{2}+\cdots+n^{2} B_{n}}{(n+1)^{2}}=O\left(n^{-\alpha}\right),
\end{aligned}
$$

by (4). But $\bar{\sigma}_{n}-\bar{f}=O\left(n^{-\alpha}\right)$ obviously implies $\bar{\sigma}_{n}^{2}-\bar{f}=O\left(n^{-\alpha}\right)$, hence $\bar{\sigma}_{n}-\bar{\sigma}_{n}^{2}$ $=O\left(n^{-\alpha}\right)$, and thus $\bar{s}_{n}-\bar{\sigma}_{n}=O\left(n^{-\alpha}\right)$. Comparing this with (2) we see that

$$
\xi_{n}(x)-\bar{f}(x)=O\left(n^{-\alpha}\right)
$$

uniformly in $x$, which was to be proved.

Suppose now that $\alpha \geqq 1$. We have, by hypothesis,

$$
R_{n}=\sum_{n}^{\infty} A_{p}=O\left(n^{-\alpha}\right)
$$

Let $\beta=\alpha-\epsilon$ where $0<\epsilon<1$. We have

$$
\sum_{n}^{\infty} A_{p} p^{\beta}=\sum_{n}^{\infty}\left(R_{p}-R_{p+1}\right) p^{\beta}=R_{n} n^{\beta}+\sum_{n+1}^{\infty} R_{p}\left[p^{\beta}-(p-1)^{\beta}\right]=O\left(n^{-}\right) .
$$

Hence, by the above proved result,

$$
S_{n}=\sum_{n}^{\infty} B_{p} p^{\beta}=\sum_{1}^{\infty} B_{p} p^{\beta}-\sum_{1}^{n-1} B_{p} p^{\beta}=O\left(n^{-c}\right),
$$

and this implies

$$
\sum_{n}^{\infty} B_{p}=\sum_{n}^{\infty} \frac{S_{p}-S_{p+1}}{p^{\beta}}=\frac{S_{n}}{n^{\beta}}+\sum_{n+1}^{\infty} S_{p}\left[\frac{1}{p^{\beta}}-\frac{1}{(p-1)^{\beta}}\right]=O\left(n^{-\alpha}\right),
$$

which completes the proof.

TheOREM IV. Let $f(x)$ and $s_{n}(x)$ have the same meaning as in Theorem III. If $f(x)-s_{n}(x)=O\left(n^{-\alpha}\right), 0<\alpha<1$, uniformly in $x$, then the derivative $s_{n}^{\prime}(x)$ is $O\left(n^{1-\alpha}\right)$ uniformly in $x$.

We have, as in the proof of Theorem III, and using the same notations: 


$$
s_{n}(x)-\sigma_{n}(x)=O\left(n^{-\alpha}\right)
$$

which implies

$$
s_{n}^{\prime}(x)-\sigma_{n}^{\prime}(x)=O\left(n^{1-\alpha}\right)
$$

But

$$
\begin{aligned}
\sigma_{n}^{\prime}(x) & =-\frac{n B_{1}+2(n-1) B_{2}+\cdots+n B_{n}}{n+1} \\
& =-(n+2)\left[\bar{\sigma}_{n}-\bar{\sigma}_{n}^{2}\right]=O\left(n^{1-\alpha}\right),
\end{aligned}
$$

since we have seen that $\bar{\sigma}_{n}-\bar{f}$ and $\bar{\sigma}_{n}^{2}-\bar{f}$ are $O\left(n^{-\alpha}\right)$. Hence also

$$
s_{n}^{\prime}(x)=O\left(n^{1-\alpha}\right) \text {. }
$$

A proof analogous to that of Theorem III shows that, if $f \in L$, and if $\int_{0}^{2 \pi}\left|f-s_{n}\right| d x=O\left(n^{-\alpha}\right)$, then $\int_{0}^{2 \pi}\left|\bar{f}-\bar{s}_{n}\right| d x=O\left(n^{-\alpha}\right)$. Instead of Bernstein's theorem on the derivatives of trigonometric polynomials we apply the corresponding result for mean values (T.S. p. 155). It may be added that for $p>1$ the inequality $\left\{\int_{0}^{2 \pi}\left|f-s_{n}\right|^{p} d x\right\}^{1 / p}=O\left(n^{-\alpha}\right)$ implies $\left\{\int_{0}^{2 \pi}\left|\bar{f}-\bar{s}_{n}\right| p d x\right\}^{1 / p}=O\left(n^{-\alpha}\right)$ and that this may be obtained by a similar argument. The result, however, is of no particular interest, being a corollary of the well known inequality $\int_{0}^{3 \pi}|\bar{g}|^{p} d x \leqq M_{p}^{p} \int_{0}^{3 \pi}|g|^{p} d x$ of M. Riesz (T.S. p. 147).

A similar extension applies to Theorem IV.

THEOREM V. Let $\sum_{0}^{\infty} A_{n}$ be any trigonometric series $\left(A_{n}=a_{n} \cos n x+b_{n} \sin n x\right)$, and let $\sum_{1}^{\infty} B_{n}$ be the conjugate series. Denote by $s_{n}$ and $\bar{s}_{n}$ the partial sums $\sum_{0}^{n} A_{k}$ and $\sum_{1}^{n} B_{k}$ respectively. Let $\alpha>0$. If $s_{n}(x)=O\left(n^{\alpha}\right)$ uniformly in $x$, then $\xi_{n}(x)$ $=O\left(n^{\alpha}\right)$ uniformly in $x$. If $\int_{0}^{2 \pi}\left|s_{n}\right| d x=O\left(n^{\alpha}\right)$, then $\int_{0}^{3_{2}}\left(\bar{s}_{n} \mid d x=O\left(n^{\alpha}\right)\right.$.

This theorem is analogous to a well known result stating that, if $z=r e^{i \theta}$, $\alpha>0$, and if $f(z)=u(r, \theta)+i v(r, \theta)$ is regular for $|z|<1$, then $u(r, \theta)=O(1-r)^{-\alpha}$ implies $v(r, \theta)=O(1-r)^{-\alpha}$ and $\int_{0}^{2 \pi}|u(r, \theta)| d \theta=O(1-r)^{-\alpha}$ implies $\int_{0}^{2 \pi}|v(r, \theta)| d t$ $=O(1-r)^{-\alpha}$ (see Hardy and Littlewood [2], Cartwright [1], or Zygmund [4]).

Suppose that

$$
\left|s_{n}(x)\right| \leqq \phi(n)
$$

for all $x$ and $n$. Then, by S. Bernstein's theorem,

$$
\left|\frac{s_{n}^{\prime}(x)}{n}\right| \leqq \phi(n) .
$$

Denoting by $\bar{\sigma}_{n}$ the Cesàro means of order 1 of $\sum_{1}^{\infty} B_{n}$, we have

$$
\left|\bar{\sigma}_{n}-\bar{s}_{n}\right|=\left|\frac{s_{n}^{\prime}(x)}{n+1}\right| \leqq \phi(n) .
$$

On the other hand, 
Hence

$$
\left|\bar{\sigma}_{n}-\bar{\sigma}_{n-1}\right|=\left|\frac{s_{n}^{\prime}(x)}{n(n+1)}\right| \leqq \frac{\phi(n)}{n+1} .
$$

$$
\left|\bar{\sigma}_{n}\right| \leqq\left|\bar{\sigma}_{1}\right|+\left|\bar{\sigma}_{2}-\bar{\sigma}_{1}\right|+\cdots+\left|\bar{\sigma}_{n}-\bar{\sigma}_{n-1}\right| \leqq \sum_{1}^{n} \frac{\phi(\nu)}{\nu+1}=O\left(n^{\alpha}\right),
$$

if $\phi(n)=O\left(n^{\alpha}\right)$. And this, compared with (5), gives $\bar{s}_{n}(x)=O\left(n^{\alpha}\right)$, the required result.

The second part of Theorem $\mathrm{V}$ is proved similarly.

Theorem $\mathrm{V}$ is a special case of the following theorem.

THEOREM VI. Let $\alpha>0, \beta>-1$. Let $\sigma_{n}{ }^{\beta}(x)$ and $\bar{\sigma}_{n}{ }^{\beta}(x)$ denote respectively the $(C, \beta)$ means of the series $\sum_{0}^{\infty} A_{k}$ and $\sum_{1}^{\infty} B_{k}$. If $\sigma_{n}^{\beta}(x)=O\left(n^{\alpha}\right)$ uniformly in $x$, then $\bar{\sigma}_{n}^{\beta}(x)=O\left(n^{\alpha}\right)$ uniformly in $x$. If $\int_{0}^{2 \pi}\left|\sigma_{n}^{\beta}(x)\right| d x=O\left(n^{\alpha}\right)$, then $\int_{0}^{2 \pi}\left|\bar{\sigma}_{n}^{\beta}(x)\right| d x=O\left(n^{\alpha}\right)$.

Let $\gamma_{n}{ }^{\beta}$ denote the Cesàro numbers, $\gamma_{n}{ }^{\beta}=(\beta+1)(\beta+2) \cdots(\beta+n) / n !$. The Cesàro sums $\sigma_{n}^{\beta}$ for any numerical series $u_{0}+u_{1}+u_{2}+\cdots$ are defined by the formula

$$
\sigma_{n}^{\beta}=\frac{1}{\gamma_{n}^{\beta}} \sum_{v=0}^{n} \gamma_{r \rightarrow,}^{\beta} u_{v} .
$$

It is easy to verify that

$$
\begin{aligned}
& \sigma_{n}^{\beta}-\sigma_{n}^{\beta+1}=\frac{1}{n+\beta+1} \frac{1}{\gamma_{n}^{\beta}} \sum_{\nu=0}^{n} \gamma_{n-\nu}^{\beta} \nu u_{v}, \\
& \sigma_{n}^{\beta}-\sigma_{n-1}^{\beta}=\frac{\beta}{n(n+\beta)} \frac{1}{\gamma_{n}^{\beta-1}} \sum_{\nu=0}^{n} \gamma_{n-\nu}^{\beta-1} \nu u_{v} .
\end{aligned}
$$

In particular, the $(C, \beta)$ means of the series $\sum_{0}^{\infty} A_{k}$ and $\sum_{1}^{\infty} B_{k}$ satisfy the equations

$$
\begin{aligned}
& \bar{\sigma}_{n}^{\beta}(x)-\bar{\sigma}_{n}^{\beta+1}(x)=-\frac{1}{n+\beta+1} \frac{d}{d x} \sigma_{n}^{\beta}(x), \\
& \bar{\sigma}_{n}^{\beta}(x)-\bar{\sigma}_{n-1}^{\beta}(x)=-\frac{\beta}{\dot{n}(n+\beta)} \frac{d}{d x} \bar{\sigma}_{n}^{\beta-1}(x) .
\end{aligned}
$$

If we use these two equations, the proof of Theorem VI becomes parallel to that of Theorem V.

It is clear that in all the results the " $O$ " may be replaced by " 0. "

\section{REFERENCES}

1. M. L. Cartwright, On analytic functions regular in the unit circle, Quart. J. Math. vol. 4 (1933) pp. 246-247. 
2. G. H. Hardy and J. E. Littlewood, Some properties of conjugate functions, J. Reine Angew. Math. vol. 167 (1932) pp. 405-423.

3. Ch. J. de la Vallée Poussin, Leçons sur l'approximation, Paris, 1919.

4. A. Zygmund, Smooth functions, Duke Math. J. vol. 12 (1945) pp. 47-76.

5. - Trigonometrical series, Warszawa-Lw6w, 1935.

Massachusetts Institute of Technology and HaRvard University, Cambridge, Mass.

Mount Holyoke College, South Hadley, Mass. 